\title{
Effect of realistic and filtered stellar photometric noise on the detection of moons using photometric transit timing
}

\author{
K. Lewis ${ }^{1}$
}

1 Monash University, Clayton 3800, Australia [karen.lewis@sci.monash.edu.au]

\begin{abstract}
The photometric transit timing technique was proposed by Szabó et al. (2006) as a method for discovering moons of transiting extrasolar planets. In the preliminary analysis of this technique, it was assumed that the noise in the transit lightcurve was well described by uncorrelated white noise. However, this assumption is not necessarily realistic. To determine the effect of using more realistic lightcurves, transit timing uncertainties are calculated for the case of white noise, measured solar photometric noise and measured solar photometric noise that has been filtered. It is found that for light curves contaminated with realistic photometric noise, the transit timing uncertainties are dramatically increased (and thus moon detection reduced). In addition, we find that while filtering reduced this problem, it did not negate it.
\end{abstract}

\section{Detecting Moons of Transiting Planets}

With over 100 planets detected, the transit technique has come of age. As a result, methods for detecting moons of these planets have been proposed. These range from directly measuring the transit of the moon (Sartoretti \& Schneider 1999), to measuring transit-to-transit perturbations in transit duration (Kipping 2009), midtime of planetary transit (Sartoretti \& Schneider 1999) and transit photocenter (Szabó et al. 2006). However, these investigations are by no means complete.

\section{Effect of Realistic Photometric Noise}

Lightcurves of the Sun, and other Sun-like stars display long terms trends (e.g. Lockwood et al. 2007). While it has long been known that this correlated noise affects the detectability of transiting planets (e.g. Borucki et al. 1985), little has been done to determine its effect on moon detection.

For this work, the effect of realistic photometric noise on the photometric transit timing method was investigated. This method was selected as:

- It has only been analysed using white (gaussian and uncorrelated) photometric noise, and

- It uses perturbations in the time of the photocenter, defined as the first moment of the photon deficit, to detect moons. Consequently it is mathematically different from transit detection. 


\section{Photometric Transit Timing}

$\tau_{j}$, the photocenter of the $j^{\text {th }}$ transit, can be written as

$$
\tau_{j}=t_{0}+j T_{p}+\Delta \tau_{j}+\epsilon_{j}
$$

where $t_{0}$ is the unperturbed time of the zeroth transit, $T_{p}$ is the planet's orbital period, and where $\Delta \tau_{j}$ and $\epsilon_{j}$ are the perturbation due to the moon and the photometric noise respectively. Thus, the process of determining if a moon could be detected by photometric transit timing, is the process of comparing $\Delta \tau_{j}$ and $\epsilon_{j}$ for a given sequence of transits.

From Simon et al. (2008) we have that

$$
\Delta \tau_{j} \approx \frac{a_{p m}}{v_{t r}}\left(\frac{R_{m}^{2}}{R_{p}^{2}}+\frac{M_{m}}{M_{p}}\right)
$$

where $a_{p m}$ is the projected distance between the planet and moon for that transit, $v_{t r}$ is the transit velocity, and where $R_{p}, M_{p}, R_{m}$ and $M_{m}$ are the radii and masses of the planet and moon respectively.

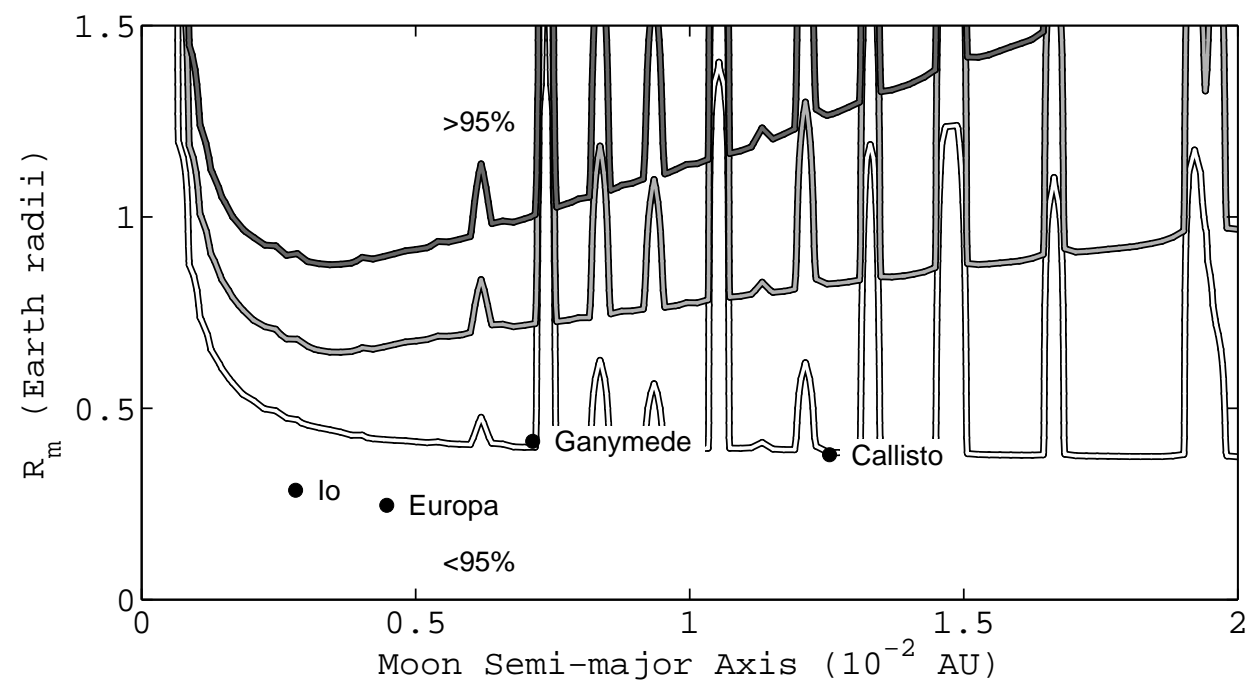

Figure 1.: 95\% detection thresholds for the case of white (white), solar (dark grey) and filtered solar (light grey) noise for a Jupiter-Ganymede planet-moon pair 0.5 AU from a Sun-like host star. For comparison, the locations of the Galilean moons are overplotted.

We investigated the behaviour of $\epsilon_{j}$ for the case of white noise, solar photometric noise and solar photometric noise filtered using the method of Lanza et al. (2003), and found that $\epsilon_{j}$ was gaussian and uncorrelated between transits for all tidally stable planet-moon pairs likely to be discovered by CoRoT and Kepler (Lewis, in preparation). 


\section{Effect of Realistic Noise on Moon Detection}

The effect of realistic photometric noise on moon detection was investigated as a function of moon size and semi-major axis. Assuming a given planetary host and moon density, equation (2) was used to create a grid of sequences of $\Delta \tau_{j}$. For each grid point, the statistical significance of detection was determined for the cases of white, solar and filtered solar noise using least squares fitting. This process was then repeated for a set of different initial moon positions. Then, the percentage of moons at each grid point which could have been detected at the 3 sigma level was determined to yield detection thresholds. An example set of thresholds is shown in figure 1. As can be seen, the effect of realistic photometric noise is to suppress moon detection, and in particular, to suppress the detection of distant moons.

\section{References}

Borucki W. J., Scargle J. D., \& Hudson H. S. 1985, ApJ, 291, 852

Kipping D. M. 2009, MNRAS, 392, 181

Lanza A. F., Rodonò M., Pagano I., Barge P., \& Llebaria A. 2003, A\&A, 403, 1135

Lewis K. M. in prep., MNRAS

Lockwood G. W., et al. 2007, ApJS, 171, 260

Sartoretti P., \& Schneider J. 1999, Ap\&SS, 134, 553

Simon A., Szátmary K., \& Szabó Gy. M. 2007, A\&A, 470, 727

Szabó Gy. M., Szátmary K., Divéki Zs., \& Simon A. 2006, A\&A, 450, 395 